\title{
Travel-time tomography in shallow water: Experimental demonstration at an ultrasonic scale
}

\author{
Philippe Roux ${ }^{\text {a) }}$ \\ Institut des Sciences de la Terre, Université Joseph Fourier, Centre National de la Recherche Scientifique, \\ Unité Mixte de Recherche 5275, 1381 Rue de la Piscine, Saint-Martin d'Hères, France \\ Ion Iturbe and Barbara Nicolas \\ Laboratoire Grenoble Images Parole Signal Automatique, Institut Polytechnique de Grenoble, Centre \\ National de la Recherche Scientifique, Unité Mixte de Recherche 5216, 961 Rue de la Houille Blanche, \\ Saint-Martin d'Hères, France

\begin{abstract}
Jean Virieux
Institut des Sciences de la Terre, Université Joseph Fourier, Centre National de la Recherche Scientifique, Unité Mixte de Recherche 5275, 1381 Rue de la Piscine, Saint-Martin d'Hères, France
\end{abstract}

\author{
Jérôme I. Mars \\ Laboratoire Grenoble Images Parole Signal Automatique, Institut Polytechnique de Grenoble, Centre \\ National de la Recherche Scientifique, Unité Mixte de Recherche 5216, 961 Rue de la Houille Blanche, \\ Saint-Martin d'Hères, France
}

(Received 8 July 2010; revised 23 June 2011; accepted 7 July 2011)

\begin{abstract}
Acoustic tomography in a shallow ultrasonic waveguide is demonstrated at the laboratory scale between two source-receiver arrays. At a 1/1 000 scale, the waveguide represents a 1.1-km-long, 52m-deep ocean acoustic channel in the kilohertz frequency range. Two coplanar arrays record the transfer matrix in the time domain of the waveguide between each pair of source-receiver transducers. A time-domain, double-beamforming algorithm is simultaneously performed on the source and receiver arrays that projects the multi-reflected acoustic echoes into an equivalent set of eigenrays, which are characterized by their travel times and their launch and arrival angles. Travel-time differences are measured for each eigenray every $0.1 \mathrm{~s}$ when a thermal plume is generated at a given location in the waveguide. Travel-time tomography inversion is then performed using two forward models based either on ray theory or on the diffraction-based sensitivity kernel. The spatially resolved range and depth inversion data confirm the feasibility of acoustic tomography in shallow water. Comparisons are made between inversion results at 1 and $3 \mathrm{MHz}$ with the inversion procedure using ray theory or the finite-frequency approach. The influence of surface fluctuations at the airwater interface is shown and discussed in the framework of shallow-water ocean tomography.
\end{abstract}

(C) 2011 Acoustical Society of America. [DOI: 10.1121/1.3621271]

PACS number(s): 43.30.Pc, 43.60.Fg, 43.60.Pt [DRD]

Pages: $1232-1241$

\section{INTRODUCTION}

Ocean acoustic tomography (OAT) was introduced by Munk and Wunsh (1979) as a remote-sensing technique for large-scale monitoring of the ocean interior using lowfrequency sound. Tomography data are strongly dependent on a uniform and dense spatial coverage of the oceanic waveguide by the ray paths between a set of sources and receivers. Taking advantage of multipath propagation to further improve the medium coverage, OAT relies on the identification and tracking of stable ray arrivals, and it uses the arrival-time changes to estimate the ocean variability. The variation of the arrival time of these rays is used to solve the inverse problem and to estimate the physical properties as sound speed variations or currents (Cornuelle et al., 1985; Worcester et al., 1985; Taniguchi et al., 2010). Following the approach of Munk, OAT became very popular in the

\footnotetext{
a) Author to whom correspondence should be addressed. Electronic mail: philippe.roux@obs.ujf-grenoble.fr
}

1990s as a way to provide rapid surveys spatially resolved in range and depth in both deep and shallow water (Munk et al., 1995; Lynch et al., 1996; Apel et al., 1997).

To identify and use a ray in the tomography process, its arrival time has to be measured accurately, and the ray has also to be assigned to a theoretical ray path. In other words, for an arrival to be useful, it must be stable enough to track over time and be unambiguously identifiable with a ray path or a ray-sampling kernel. A good background model of the propagation medium is then essential.

Furthermore, as well as such experimental limitations, Munk and Wunsch (1983) discussed theoretically the number of resolved eigenrays or eigenmodes from a point-topoint, deep-water experimental configuration where the travel-time separation between low-grazing angle rays was subject to bandwidth issues. Rodriguez and Jesus (2000) pursued the same analysis in shallow water, showing the physical limitations of travel-time tomography.

Finally, in both deep and shallow waters, the promising range-depth images of sound-speed fluctuations initially 
expected from OAT have been a difficult target because of (1) technical limitations associated with the deployment of a large number of sources and receivers at sea and (2) the limited number of resolved rays from a point-to-point approach in a multipath environment.

The difficulties associated with point (2) in the preceding text can be eased when the classical point-to-point configuration is replaced by an array of sources and receivers and with the use of array processing. In a recent study, Roux et al. (2008) developed a double-beamforming (DBF) algorithm in which time-delay beamforming is performed on both receiving and emitting arrays; this allows more robust ray-path identification through estimation of their launch angle, receiver angle, and arrival time. The DBF algorithm thus reduces the dependence on the background model. This DBF approach also tackles the classical problem of resolved/ unresolved eigenrays in a point-to-point or point-to-array configuration. Basically, every acoustic ray can now be isolated and identified, given the diffraction limit of the system, and depending on the size, central frequency, and bandwidth of the arrays. Finally, DBF naturally increases the signal-tonoise ratio as array gain is performed on both source and receiver arrays. In their study, Roux et al. (2008) discussed the suitability of the signals observed after DBF as estimators of ray travel times.

DBF was implemented in shallow-water configurations with numerical and experimental data. First, DBF was shown using simulation results to provide reliable travel-time measurements from which successful tomography can be performed in shallow water (Iturbe et al., 2009a). Second, stable ray identification was performed through DBF with shallow-water ocean data to monitor the evolution of eigenrays that propagate in the waveguide for an extended period of time. Indeed, to avoid technical limitations and environmental uncertainties of large-scale, deep-water experiments (see North Pacific Acoustic Laboratory), smaller-scale Focused Acoustic Field (FAF) experiments (FAF03 and FAF05 in 2003 and 2005) were conducted, with ranges from 1 to $8 \mathrm{~km}$, in a 50 - to 100 -m-deep waveguide and with acoustic signals centered at $3 \mathrm{kHz}$, [see Roux et al. (2004) for details].

One of the goals of these FAF experiments was to demonstrate the feasibility of shallow-water tomography between two vertical arrays of sources and receivers that covered a large portion of the water column. However, despite a total number of more than 1000 eigenrays extracted and identified between the two arrays, the travel-time data analysis revealed three major difficulties. First, the rays that hit the dynamic ocean surface several times are polluted by fast-evolving, random, travel-time fluctuations that are comparable to the slow-evolving travel-time changes expected from sound-speed fluctuations in the water column. Second, the similarity between the ray paths refracted at the thermoclyne means that these travel-time measurements are strongly correlated, while uncorrelated measurements would provide more information about the spatial dependence of sound-speed fluctuations. Last, some acoustic rays were not continuously tracked over the period of time under investigation (on the order of a few hours), which suggests the presence of micro-pathing associated with the time-evolving and range-dependent sound-speed fluctuations at the thermoclyne.

Pursuing our shallow-water tomography goal, it was clear that new approaches were needed with better control of all of the required ingredients for a spatially resolved tomography experiment; i.e., of (1) the acquisition setup involving source and receiver arrays; (2) the appropriate background model for the shallow-water propagation medium; and (3) the time-evolving fluctuations of the acoustic environment.

One solution was to experimentally "revisit" the shallow-water tomography problem through a laboratory experiment at the ultrasonic scale. Here, the use of multi-channel electronics connected to two 64-element source and receiver arrays provides the complete acquisition of the transfer function of the ultrasonic waveguide in the time-domain at a rate of $100 \mathrm{~ms}$ (10 scans of the waveguide per second). This acquisition system perfectly matches the versatile model-tank facilities (Fig. 1) in which small-scale waveguide experiments are performed and controlled in an ultrasonic regime from 0.5 to $5 \mathrm{MHz}$. This laboratory setup actually works as an analog computer for ultrasonic propagation in close connection with shallow-water ocean physics.

The objectives of this study are threefold. First, the feasibility of travel-time tomography is demonstrated in a scaled-down ultrasonic demonstrator of a shallow-water ocean environment. Second, the range-depth resolution scale that is obtained with experimental data at the two frequencies 1 and $3 \mathrm{MHz}$ is discussed using a forward model that is based on either ray theory or diffraction-based physics. Last, limitations of OAT in the ocean are investigated that arise from the experimental surface fluctuations that are observed at the ultrasonic scale.

The structure of this study is as follows. Section II deals with the ultrasonic data that are obtained in the waveguide at the laboratory scale. More than 1000 eigenrays are identified, and their travel times are extracted using array processing on the source-receiver arrays. Section III describes the travel-time tomography approach through ray theory and through calculation of the diffraction-based sensitivity kernel. Dynamic, spatially resolved, tomography results are

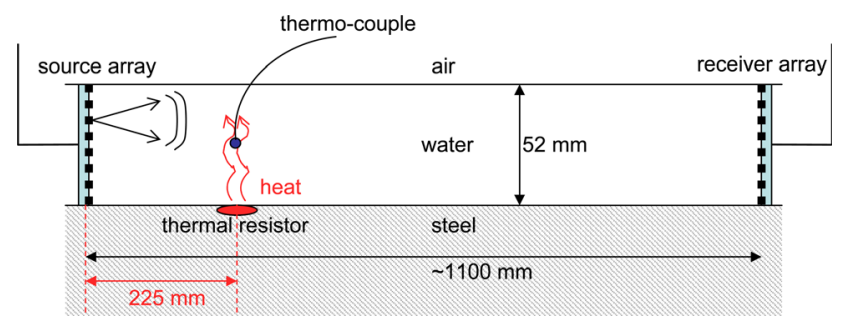

FIG. 1. (Color online) Schematic diagram of the experimental setup. Two coplanar, 64-element, source-receiver arrays centered at 1 or $3 \mathrm{MHz}$ face each other in an acoustic waveguide that is delimited by two air-water and water-steel interfaces. The sound speed is uniform in the waveguide. The sound-speed variations are generated by a thermal resistor that is embedded in the bottom of the ultrasonic waveguide (range, $225 \mathrm{~mm}$ ). During the 40s-long acoustic acquisition, the heating system is activated at acquisition time $\tilde{t}=T_{0}$ and stopped at $\tilde{t}=T_{0}+T$. In a separate experiment, a thermocouple was positioned variously above the resistor to measure the temperature of the thermal plume during the same heating process. 
presented in Sec. IV, and the laboratory-scaled data are finally discussed in the framework of ocean acoustic research in Sec. V.

\section{SHALLOW WATER ACOUSTICS AT THE ULTRASONIC SCALE: THE EXPERIMENTAL SETUP}

Figure 1 illustrates the experimental setup. Two coplanar vertical arrays of 64 transducers face each other in a $\sim 1.10$-m-long, 5.4-cm-deep ultrasonic waveguide. The central frequency of the transducers is 1 or $3 \mathrm{MHz}$ (wavelength $\lambda=1.5$ or $0.5 \mathrm{~mm}$, respectively) with a $50 \%$ frequency bandwidth. At both 1 and $3 \mathrm{MHz}$, each transducer size is 0.75 $\mathrm{mm} \times 12 \mathrm{~mm}$, which makes the linear arrays relatively omni-directional in the plane defined by the source-receiver arrays and very collimated outside of this plane (to prevent acoustic echoes from the sidewalls of the tank). The waveguide bottom is made of steel for which the boundary conditions are nearly perfect at the water-bottom interface. Surface waves can be added at the air-water interface during the experiment. In a water tank, surface waves are capillarygravity waves with typical spatial wavelengths on the order of a few centimeters that scale with the ultrasonic wavelength in the same way that gravity-wave wavelengths scale at sea with acoustic wavelengths in the kilohertz range.

The acquisition sequence consists of recording the whole transfer matrix between each source and each receiver in the time domain. This is performed through a round-robin sequence, during which each source successively emits a broadband 2- $\mu$ s-long (respectively, 1- $\mu$ s-long) ultrasonic pulse at the $1 \mathrm{MHz}$ (respectively, $3 \mathrm{MHz}$ ) central frequency of the transducers (Roux et al., 2004). The duration between the pulses emitted from each source is chosen to be no longer than the maximum time spread due to multipath $(\sim 100 \mu \mathrm{s})$, which provides the full waveguide transfer matrix in less than $100 \mathrm{~ms}$. At the ultrasonic scale, this repetition time of the acquisitions is short enough to follow the dynamic behavior of the waveguide caused by surface waves for example.

The waveguide characteristics (depth and range) and transducers directivity provide approximately 12 reflected acoustic echoes between the source-receiver arrays. A 4-mm-wide, 35- $\Omega$ thermal resistor is embedded in the steel bottom (Fig. 1). When a continuous $20-\mathrm{V}$ tension is applied to the resistor, the temperature increase measured by a thermocouple in the water column rapidly reaches a maximum of $+3.5^{\circ} \mathrm{C}$, which should introduce a local positive soundspeed change on the order of $15 \mathrm{~m} / \mathrm{s}$.

Using this experimental setup, the tomography experiment was run as follows. At $t=0 \mathrm{~s}$, the multi-channel acquisition between the two arrays was started, which results in successive recordings of transfer matrices made up of 64 sources $\times 64$ receivers $=4096$ ultrasonic signals every 100 $\mathrm{ms}$. In the following, to avoid confusion, clear distinction is made between the time $t$ (in $\mu \mathrm{s}$ ) of the acoustic signals sampled at $80 \mathrm{MHz}$, and the time of each acquisition of the waveguide transfer matrix (called the acquisition time $\tilde{t}$, in s) at the rate of $10 \mathrm{~Hz}$ (one acquisition every $100 \mathrm{~ms}$ for the whole matrix).

Between $\tilde{t}=T_{0}$ and $\tilde{t}=T_{0}+T$, a $20-\mathrm{V}$ tension was applied to the thermal resistor. In practice, $T_{0}=2.5 \mathrm{~s}$ and
$T=15 \mathrm{~s}$ were used at $3 \mathrm{MHz}$ and $T_{0}=7.5 \mathrm{~s}$ and $T=20 \mathrm{~s}$ at $1 \mathrm{MHz}$. The multi-channel acquisition was finally stopped at $\tilde{t}=40 \mathrm{~s}$ for a total recording of 400 acoustic-transfer matrices from which the spatial and temporal sound-speed fluctuations caused by the local thermal plume generated from the bottom are inverted in the ultrasonic waveguide.

Figures 2(a) and 2(b) show a depth-versus-time representation of the ultrasonic field arising from one source and received on the whole receiver array after propagation through the waveguide at 3 and $1 \mathrm{MHz}$, respectively. At both frequencies, the pressure field is made up of several acoustic wavefronts that result from reflection of the emitted pulse on the surface and bottom of the ultrasonic waveguide. Such an acoustic field at a small scale clearly resembles shallow-water data recorded at sea with a similar scale ratio among range, depth, and acoustic wavelength (Roux et al., 2008). For one source-receiver pair, Figs. 2(c) and 2(d) show the time evolution of one acoustic echo arrival during the 400 successive acquisitions during the creation of the thermal plume from the bottom of the waveguide. The travel-time change arising from the temperature increase is clearly observed at both 3 and $1 \mathrm{MHz}$. As expected, the resolution is greater at the higher frequency. On the other hand, some travel-time fluctuations also appear to pre-exist at 3 $\mathrm{MHz}$ before the heating is started $(\tilde{t}<5 \mathrm{~s})$, which are due to surface waves generated by a hydraulic pump used to filter the tank water [Fig. 2(c)]. These travel-time fluctuations appear negligible at $1 \mathrm{MHz}$ [Fig. 2(d)].

Time-delay beamforming is applied simultaneously at the source and receiver arrays to extract a set of eigenrays from the waveguide transfer matrix that are identified by their travel times and launch and arrival angles $\left[\theta_{r}, \theta_{s}\right]$. This DBF algorithm was recently introduced in shallow-water physics as a way to unambiguously match broadband experimental data to theoretical ray paths (Roux et al., 2008). Equation (1) presents the mathematical formulation in the time domain of this DBF processing:

$$
\begin{aligned}
P_{\mathrm{DBF}}\left(t, \theta_{r}, \theta_{s}\right)= & \frac{1}{N_{r} N_{s}} \sum_{l=1}^{N_{r}} \sum_{m=1}^{N_{s}} P\left(t+\tau\left(\theta_{r}, z_{r_{l}}\right)\right. \\
& \left.+\tau\left(\theta_{s}, z_{s_{m}}\right), z_{r_{l}}, z_{s_{m}}\right)
\end{aligned}
$$

where $z_{r_{l}}$ and $z_{S_{m}}$ are the receiver and source depths for the $N_{r}$ receivers $\left(l \in\left[1, N_{r}\right]\right)$ and $N_{s}$ sources $\left(m \in\left[1, N_{s}\right]\right)$, respectively. $\tau(\theta, z)$ corresponds to the time delay to be applied to one array element at depth $z$ to beamform in a direction $\theta$. If the sound speed is uniform $[c(z)=c]$ along the array, plane wave beamforming is obtained by

$$
\tau(\theta, \mathbf{z})=\left(z-z_{0}\right) \sin \theta / c,
$$

where $z_{0}$ is the center of the array on which the time-delay beamforming is performed. With a depth-dependent soundspeed profile, the optimal time-delay beamforming is obtained by the turning-point filter approach (Dzieciuch et al., 2001). To obtain a large number of eigenrays, DBF is performed from subarrays on both the source and receiver sides. For example, using a limited set of 11 subarrays for a total of $11 \times 11=121$ source-receiver subarray center 

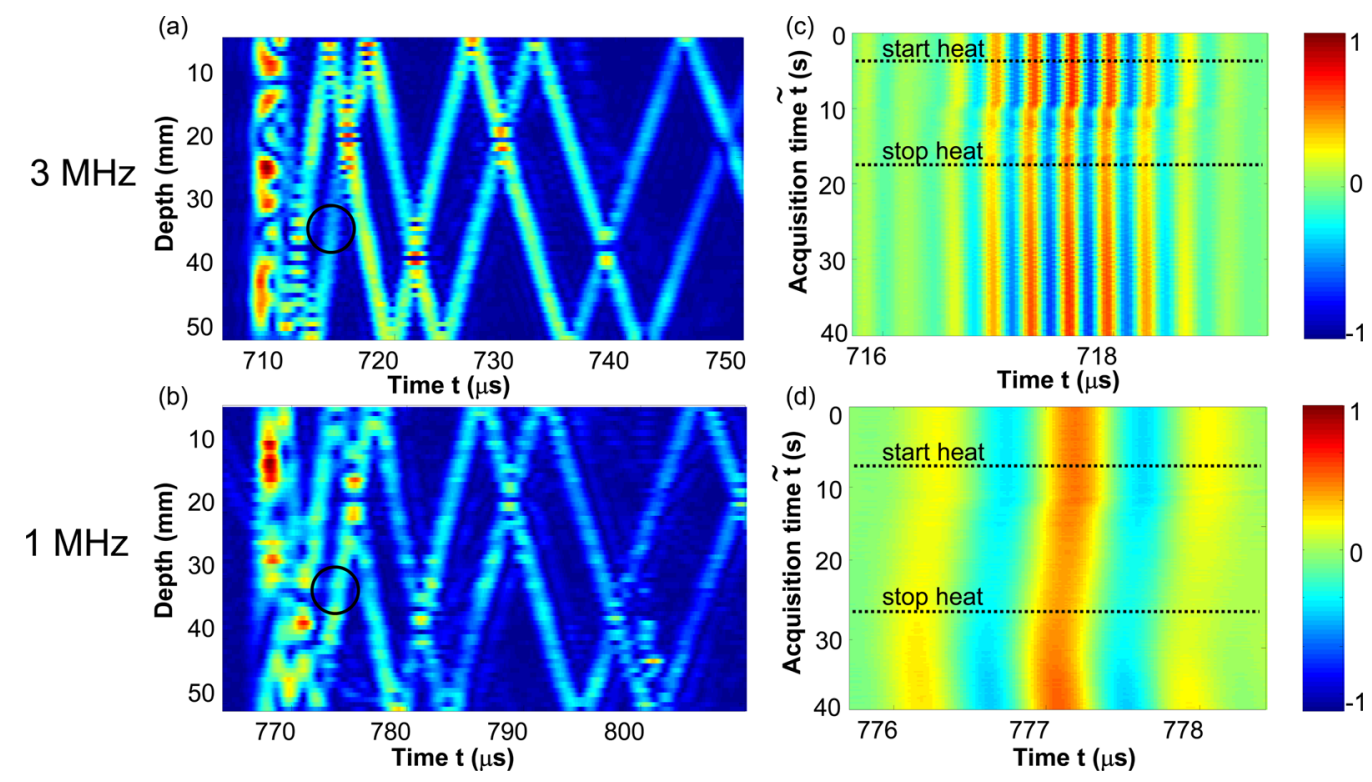

FIG. 2. (Color online) (a) and (b) Envelope of the depth versus time ultrasonic signal recorded on the receiver array for a source depth at 34 mm and a pulselike emitted signal at $3 \mathrm{MHz}$ (a) and at $1 \mathrm{MHz}$ (b). The time-axis difference in (a) and (b) is due to the different source-receiver ranges (1.05 $\mathrm{m}$ at $3 \mathrm{MHz}$ and $1.15 \mathrm{~m}$ at $1 \mathrm{MHz}$ ) in the two experiments. The pressure field is normalized relative to its maximum, and the colorbar scale is linear. Despite the slightly different ranges between the two experiments, the wavefront arrival shows the same structure at both frequencies after propagation in the ultrasonic waveguide. (c) and (d) Temporal evolution of a 2.5- $\mu$ s-long acoustic echo [receiver depth, $36 \mathrm{~mm}$, centered at the middle of the black circles in (a) and (b)] at $3 \mathrm{MHz}$ (c) and $1 \mathrm{MHz}(\mathrm{d})$ during the heating process; this shows the travel-time shift of the signals due to the local increase in sound speed. The horizontal dashed lines indicate the start and the end of the heating process.

configurations at depths $\left[z_{s 0}, z_{r 0}\right]$, more than 1400 eigenrays can finally be extracted and identified for complete cover of the plane defined by the two vertical arrays. The time evolution of an eigenray with one single bounce on the bottom is shown in Fig. 3(b). At $3 \mathrm{MHz}$, the signature of the local temperature rise on this acoustic path is clearly seen after $\tilde{t}=5 \mathrm{~s}$ by a negative travel-time change. Note that the maximal travel-time fluctuation is limited to $5 \%$ of an acoustic period.

\section{INVERSION METHOD}

As with any inversion process, travel-time tomography requires a forward model with sufficient accuracy to predict the travel times between the sources and receivers in the acoustic waveguide. Such a model is easily available in an ultrasonic waveguide where all of the parameters (i.e., range, depth of source and receiver positions, bottom properties) are known to high accuracy. However, these parameters are mostly unknown in realistic shallow-water oceanic environments; this greatly complicates the tomography task. One way to get around this major difficulty is to perform differential tomography, where the inversions to travel-time differences are limited between successive experiments. In other words, differential tomography only deals with sound-speed perturbations that are associated with travel-time fluctuations relative to an initial state that is defined as the first transfer matrix (at $\tilde{t}=0$ ) or eventually to the average transfer matrix over the whole of the experiment.

Forward modeling is performed from either ray theory or the diffraction-based sensitivity kernel to investigate the optimal resolution scale in shallow-water tomography with a finite-frequency approach. To date, the resolution limit of travel-time tomography has been studied from various aspects. These investigations have essentially relied on the specific, maybe paradoxical, nature of travel times as extracted from time-series recordings. Once picked, travel times lose the frequency information of the time series. For example, choosing times from high-frequency impulsive signals or from broadband, low-frequency signals will certainly have an impact on the tomographic resolution. However, the frequency information is not used in the travel-time tomography processing based on ray theory. In seismology, for example, ad hoc procedures for introducing frequency information have been designed with the so-called fat ray concept (Tromp et al., 2005). A more physical concept of the wave path, with relation to the wave-propagation properties, was introduced by Woodward (1992) that is closely related to Fresnel tomography in optics. In recent years, this finitefrequency influence has been investigated in underwater acoustics (Skarsoulis and Cornuelle, 2004), which suggested that higher-resolution images can be obtained from this improved description of wave-propagation physics.

From point-to-point measurements, the travel-time fluctuation $\Delta \tau$ can then be modeled through ray theory [Eq. (3)] or using the diffraction-based travel-time sensitivity kernel [TSK; Eq. (4) as

$$
\Delta \tau_{\text {ray }}=-\int_{\Gamma} \frac{\Delta c(\vec{r})}{c_{0}^{2}(\vec{r})} d s
$$

and

$$
\Delta \tau_{\mathrm{TSK}}=\int_{V} K\left(\vec{r}, \vec{r}_{s}, \vec{r}_{r}\right) \Delta c(\vec{r}) d V .
$$

In Eq. (3), $c_{0}(\vec{r})$ is the background model at the initial state, $d s$ is the curvilinear distance along the ray path $\Gamma$ connecting 

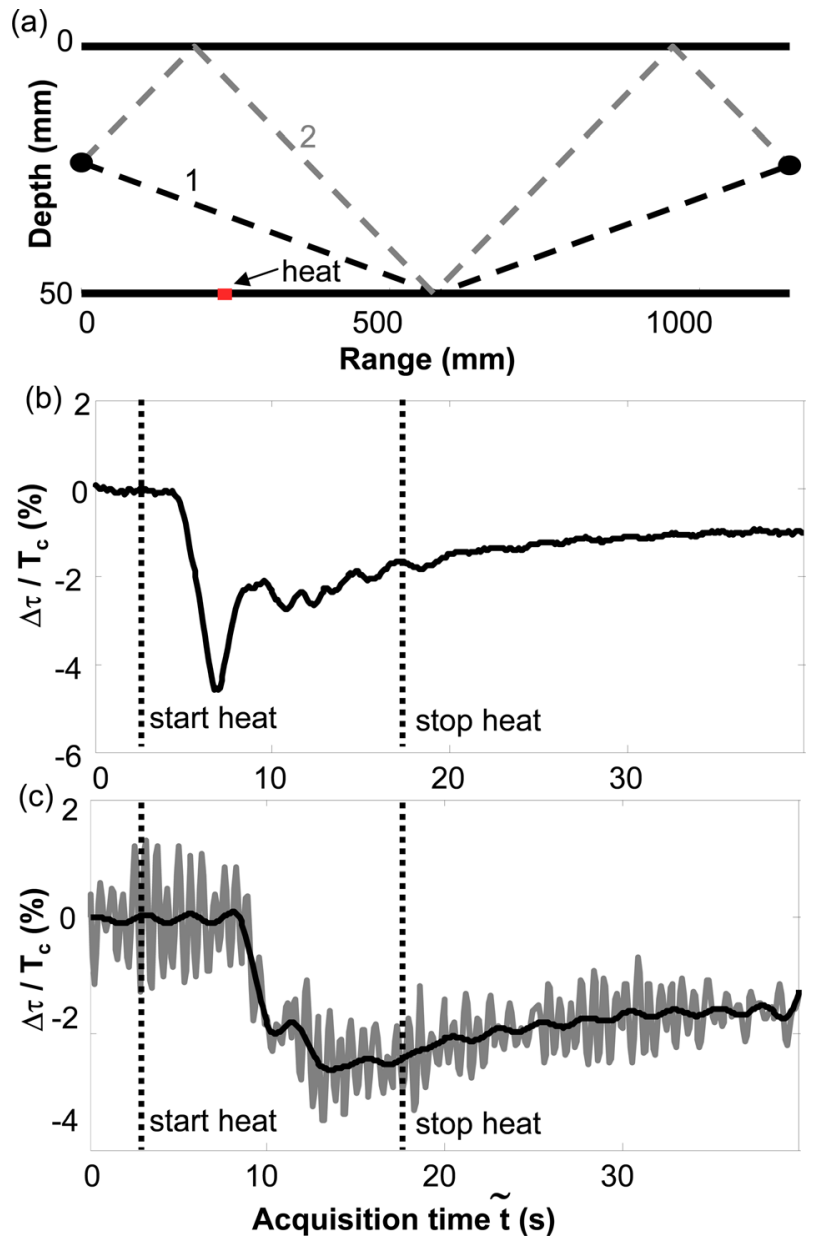

FIG. 3. (Color online) Influence of surface waves at the air-water interface on the travel-time measurements at $3 \mathrm{MHz}$ during the heating cycle for two DBF eigenrays. (a) Schematic diagram of the waveguide with planar interfaces (bold lines), the center of the source and receiver sub-arrays (full circles at each end), and the two selected eigenray paths [dashed lines, 1 (black) and 2 (gray)]. (b) Travel-time measurements after DBF for eigenray 1 (one bottom reflection) during the heating cycle. (c) Travel-time measurements (gray line) after DBF for eigenray 2 (one bottom reflection, two surface reflections) during the heating cycle. The black line corresponds to the lowpass filtered version of the gray line. Surface scattering induces travel-time fluctuations of the same order of magnitude as the thermal plume. The convection phenomenon in the volume and the surface-induced fluctuations can be studied separately because they have different characteristic times. The thermal resistance is located at a range of $225 \mathrm{~mm}$ as indicated by the black arrow (heat) in (a). The dotted lines in (b) and (c) indicate the start and the end of the heating process.

the source-receiver pair. In Eq. (4), the TSK $K\left(\vec{r}, \vec{r}_{s}, \vec{r}_{r}\right)$ between the source in $\vec{r}_{s}$ and the receiver in $\vec{r}_{r}$ is integrated over the whole volume of the waveguide $V$ (Iturbe et al., 2009b) with:

$$
\begin{aligned}
K\left(\vec{r}, \vec{r}_{s}, \vec{r}_{r}\right)= & \frac{1}{\pi} \int \frac{j \omega}{\ddot{p}_{\tau}} \frac{\omega^{2}}{c^{3}(\vec{r})} \\
& \times G\left(\vec{r}, \vec{r}_{s}, \omega\right) G\left(\vec{r}_{r}, \vec{r}, \omega\right) P_{S}(\omega) e^{j \omega \tau} d \omega .
\end{aligned}
$$

In Eq. (5), $G\left(\vec{r}_{2}, \vec{r}_{1}, \omega\right)$ corresponds to the Green's function between a source in $\vec{r}_{1}$ and a receiver in $\vec{r}_{2}, P_{S}(\omega)$ is the source spectrum, $\tau$ is the travel time of the acoustic echo between the source and the receiver, and $\ddot{p}_{\tau}$ is the secondorder time derivative of the pressure field at time $\tau$. Note that the $K$ kernel in Eq. (5) goes back to a shape that is compatible with Eq. (3) as the frequency goes to infinity (Dahlen et al., 2000).

When travel-times are extracted from DBF processing, the travel-time perturbations $\Delta \tau_{\mathrm{TSK}-\mathrm{DBF}}$ follow an equation equivalent to Eq. (4), with a TSK $K_{\mathrm{DBF}}\left(\vec{r}, \vec{r}_{s_{0}}, \vec{r}_{r_{0}}\right)$ that takes into account the DBF processing around the sub-array centers in $\left[\vec{r}_{s_{0}}, \vec{r}_{r_{0}}\right]$ (Iturbe et al., 2009b).

Very few studies have been carried out to date on ocean tomography using a diffraction-based sensitivity kernel. What makes TSK difficult to use as a forward model in shallow-water configurations is the computational cost associated with the calculation in the whole volume, as is indicated by Eq. (5), while ray-path calculations along lines are straightforward. However, in a recent study, Iturbe et al. (2009b) showed that in the context of DBF processing, the spatial shape of the TSK can be approximated for each eigenray as the product of the diffraction patterns of the source and receiver subarrays projected along the ray path. On the other hand, this approximation may not be valid for the calculation of the TSK at the waveguide interfaces where interferences and reflection coefficients have to be taken into account [see Fig. 1 of Iturbe et al. (2009b)].

Figure 4(b) shows the spatial shape of the diffractionbased TSK after DBF for one eigenray in the waveguide that can be compared with the infinite-frequency-associated ray path in Fig. 4(a). Contrary to the point-to-point TSK that classically shows a banana/doughnut shape with zero sensitivity along the ray path (Marquering et al., 1999), the TSK calculated after DBF shows maximal sensitivity on the acoustic ray. Moving from point to point to DBF analysis is equivalent to moving from Fresnel optics to Fraunhofer optics in diffraction theory. The size of the Fresnel zone is directly related to the central frequency of the acoustic signal. A higher frequency means a smaller Fresnel zone, which should provide better spatial resolution. As a comparison with Fig. 4(a), the spatial shape of the ray-based kernel is independent of the frequency, although the width of the Fresnel zone in Fig. 3(b) confirms that ray theory should no longer be valid at low frequency.

One of the goals of the present study is to show experimental tomography results performed with a forward model based on ray theory and TSK at low and high frequencies.

As carried out classically in ocean tomography, the inversion for travel-time perturbations is linearized as

$$
\Delta \tau=\mathbf{G}_{0} \Delta c+b,
$$

where $\Delta \tau$ is the data vector that is composed of travel-time perturbations extracted from the maximum number of wellidentified eigenrays after DBF, $\Delta c$ is the unknown vector parameterized as sound-speed fluctuations in the waveguide, $b$ is the noise (assumed to be Gaussian) in the travel-time measurements, and $\mathbf{G}_{\mathbf{0}}$ is the sensitivity matrix (also known as the matrix of Fréchet derivatives) that is built from Eq. (3) or (4), depending on the forward model. The matrix representation in Eq. (6) assumes that sound-speed fluctuations are discretized on a spatial representation of the waveguide. There are different appropriate bases upon which to perform this projection, such as elementary cells, sinusoidal 


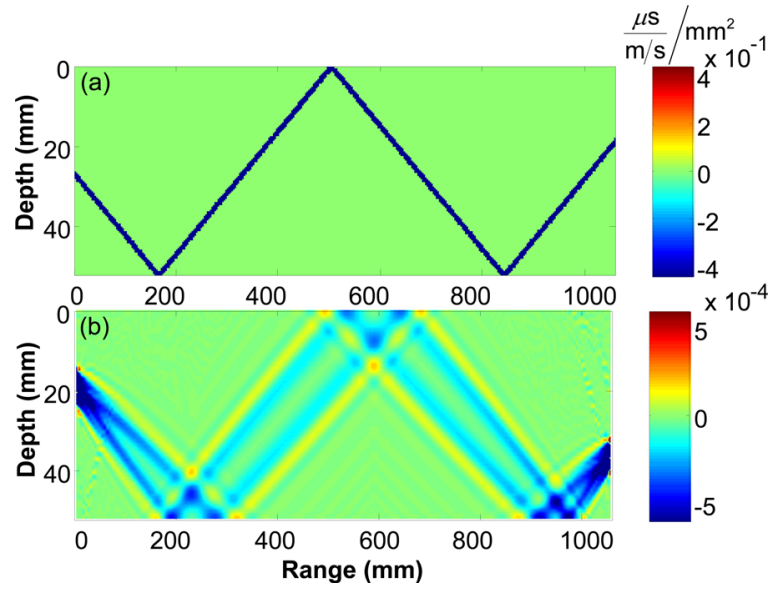

FIG. 4. (Color online) Spatial representation of the TSK for one selected eigenray after DBF, for the ray-based forward model (a) and the diffractionbased forward model (b), at $3 \mathrm{MHz}$. The colorbar scale corresponds to travel-time changes in microseconds for a sound-speed change of $1 \mathrm{~m} / \mathrm{s}$ over a $1 \mathrm{~mm}^{2}$ surface. The Fresnel zone is clearly wide in (b); this confirms that the ray approach in (a) may not be valid in this ultrasonic waveguide.

functions, and wavelets, or eventually empirical orthogonal functions deduced from a priori observations that usually allow the number of unknowns to be significantly reduced. Because no a priori information is available in our ultrasonic tank, the sound-speed fluctuations is projected in the waveguide on a set of $Q$ elementary cells in the range and depth $\left[r_{i}, z_{i}\right]$, with $i \in[1, Q]$.

The Bayesian approach for tomography has been widely discussed in underwater acoustics (Rajan et al., 1987; Battle et al., 2004) with a reference work on the general inverse problem published by Tarantola (1984). In the Bayesian approach, the general inverse problem is solved by finding the maximum a posteriori solution. If uncertainties have Gaussian distributions, the optimization problem is completely defined by second-order statistics. The maximization of the a posteriori probability density is equivalent to the minimization of the least-squares objective function $S$, expressed as

$$
S=\left(\Delta \tau-\mathbf{G}_{\mathbf{0}} \Delta c\right)^{T} \mathbf{C}_{\mathbf{d}}^{-\mathbf{1}}\left(\Delta \tau-\mathbf{G}_{\mathbf{0}} \Delta c\right)+\Delta c^{T} \mathbf{C}_{\mathbf{m}}^{-\mathbf{1}} \Delta c .
$$

In Eq. (7), it is first assumed that the average sound-speed fluctuations should be zero. The covariance matrix for the data space $\mathbf{C}_{\mathbf{d}}=\sigma_{b}^{2} \mathbf{I}$ is taken as a diagonal matrix with a uniform uncertainty equal to the noise variance $\sigma_{b}^{2}$. The a priori covariance matrix for the model space $\mathbf{C}_{\mathbf{m}}$ is also defined with matrix elements $\mathbf{C}_{\mathbf{m} i j}$ (with $i, j \in[1, Q]$ ), such that

$$
\mathbf{C}_{\mathbf{m} i j}=\sigma_{m}^{2} \exp \left(-1 / 2\left[\left(\frac{r_{i}-r_{j}}{\lambda_{r}}\right)^{2}+\left(\frac{z_{i}-z_{j}}{\lambda_{z}}\right)^{2}\right]\right) \text {. }
$$

In Eq. (8), $\sigma_{m}^{2}$ depends on the model uncertainty, and $\lambda_{r}$ and $\lambda_{z}$ represent characteristic correlation length scales in range and depth for the ultrasonic field in the waveguide. Dealing with two vertical arrays, diffraction laws state that $\lambda_{\mathrm{r}} \gg \lambda_{\mathrm{z}}$ $\sim$ a few $\lambda$ where $\lambda$ is the ultrasonic wavelength. In practice, the objective function $S$ is evaluated for different values of $\sigma_{m}, \lambda_{r}$, and $\lambda_{z}$. Increasing correlation lengths make the model smoother, thus decreasing the second terms in Eq. (7), although too much smoothness finally degrades the fit with the travel-time data, which increases the first term in Eq. (7). An optimal solution is found for $\sigma_{m}=1 \mathrm{~m} / \mathrm{s}, \lambda_{r}=37 \mathrm{~mm}$, and $\lambda_{z}=5 \mathrm{~mm}$ (Iturbe et al., 2009a).

For the minimum of the objective function $\mathrm{S}$, the inverse operator can be obtained analytically (Tarantola, 1987) by

$$
\widetilde{\mathbf{G}_{\mathbf{0}}^{-1}}=\mathbf{C}_{\mathbf{m}} \mathbf{G}_{\mathbf{0}}^{\mathbf{T}}\left(\mathbf{G}_{\mathbf{0}} \mathbf{C}_{\mathbf{m}} \mathbf{G}_{\mathbf{0}}^{\mathbf{T}}+\mathbf{C}_{\mathbf{d}}\right)^{-1}
$$

as long as the covariance matrices can be inverted. The maximum a posteriori solution is then given by

$$
\widetilde{\Delta c}=\widetilde{\mathbf{G}_{0}^{-1}} \Delta \tau
$$

\section{EXPERIMENTAL RESULTS}

The experimental tomography data obtained at $3 \mathrm{MHz}$ with TSK are presented in Fig. 5, with the 12 panels corresponding to different times during the 40-s-long recording time window. A 97\% variance reconstruction is obtained for the travel-time fluctuations for each of the 400 ultrasonic acquisitions made in the waveguide. The local heating is clearly observed at the position of the resistor in the waveguide. The dynamics of the heating process are also revealed as a thermal plume that starts at the waveguide bottom (at $\tilde{t}>5 \mathrm{~s}$ ) and moves up to the water-air interface, where it accumulates until the heating stops (at $\tilde{t}=17.5 \mathrm{~s}$ ); after this, the heat spot spreads and slowly vanishes. The set of panels in Fig. 5 illustrates this time-evolving convection phenomenon where the dynamics are slow enough to be imaged by the repetition rate of the acoustic acquisition. The time evolution of the acoustic measurements is further confirmed in a separate experiment with an equivalent heating cycle (starting at $T_{0}=2.5 \mathrm{~s}$ ), with temperature measurements using a thermocouple at different heights in the waveguide [Fig. 6(a)]. In particular, the rise times of the thermal plume from the bottom to the top of the waveguide are identical across the acoustic and thermocouple measurements [Fig. 6(b)].

The experimental results in Figs. 5 and 6 lead to the following comments. On the one hand, it is remarkable that the spatial and temporal characteristics of the heating phenomenon are well reconstructed. Following the OAT attempts in the 1980s (Cornuelle et al., 1985; Chiu and Desaubies, 1987), these results show evidence of a time-evolving and spatially resolved (both in range and depth) tomography inversion in an acoustic waveguide.

On the other hand, two flaws in the inversion results should be noted. The first concerns the tomography result at the bottom just above the resistor. The thermocouple reveals an average $3.5^{\circ} \mathrm{C}$ heating maximum at this location; this should induce a maximum sound-speed perturbation that is clearly not observed in the acoustic inversion [Fig. 6(b)]. One explanation may be the incorrect calculation of the TSK after DBF at the waveguide bottom [Fig. 4(b)], where the boundary conditions (hard bottom) are different from the pressure-release boundary conditions encountered at the airwater interface. Note that for the sake of computation time, 

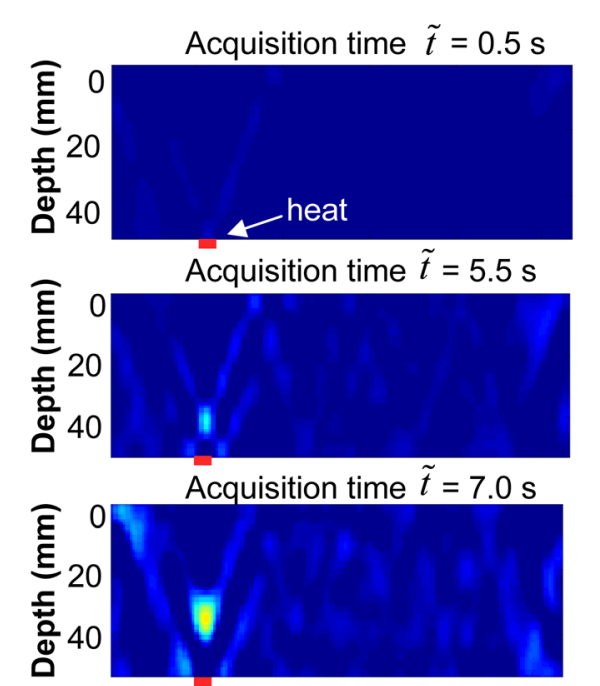

Acquisition time $\tilde{t}=8.5 \mathrm{~s}$

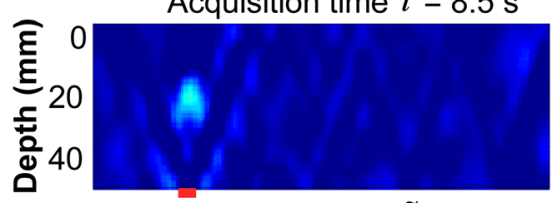

Acquisition time $\tilde{t}=10 \mathrm{~s}$
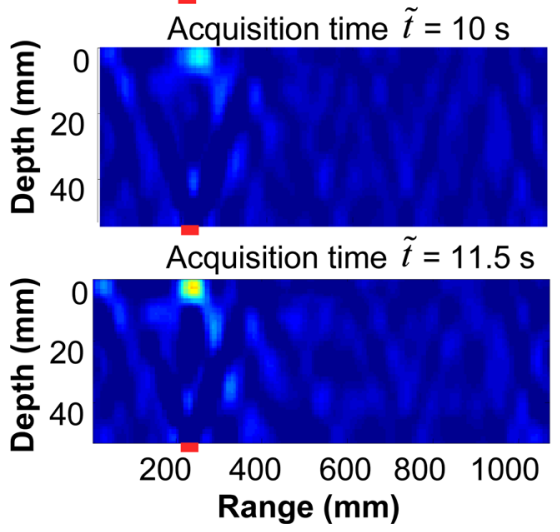

Acquisition time $\tilde{t}=13 \mathrm{~s} \quad \Delta \mathrm{c}(\mathrm{m} / \mathrm{s})$

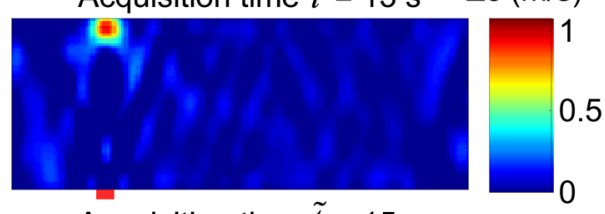

Acquisition time $\tilde{t}=15 \mathrm{~s}$

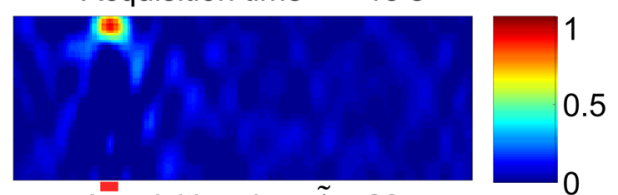

Acquisition time $\tilde{t}=20 \mathrm{~s}$

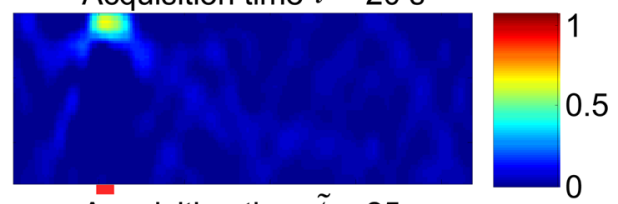

Acquisition time $\tilde{t}=25 \mathrm{~s}$

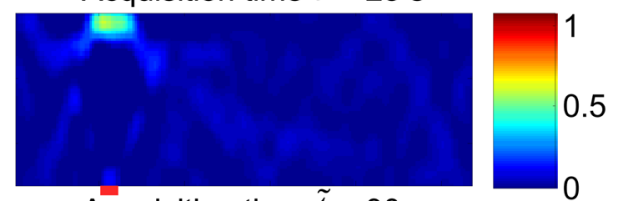

Acquisition time $\tilde{t}=30 \mathrm{~s}$

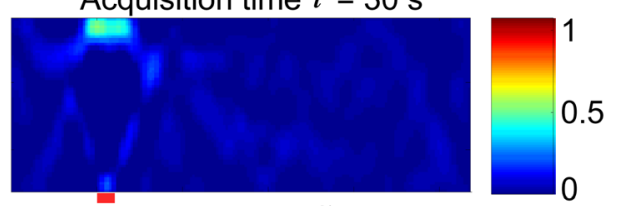

Acquisition time $\tilde{t}=35 \mathrm{~s}$

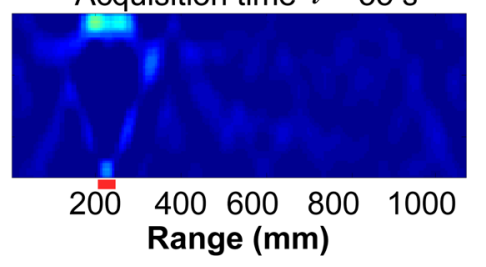

FIG. 5. (Color online) Travel-time tomography data in the ultrasonic waveguide, showing the estimated sound-speed variations (in $\mathrm{m} / \mathrm{s}$ ) using diffraction-based TSK at $3 \mathrm{MHz}$ and with DBF. The acquisition time increases from top to bottom and then from left to right. White arrow (heat), top left panel, the thermal resistance is located at a range of $225 \mathrm{~mm}$. The heating process starts at $T_{0}=2.5 \mathrm{~s}$ and stops at $T_{0}+T=17.5 \mathrm{~s}$. the TSK was calculated for each eigenray from the product of the diffraction pattern of the source-receiver arrays; this does not account for interferences and reflection coefficients at the waveguide bottom.

The second flaw in the inversion results concerns the amplitude of the estimated sound-speed fluctuations $(\sim 1$ $\mathrm{m} / \mathrm{s}$ ), as this is clearly below the expected sound-speed change $(\sim 15 \mathrm{~m} / \mathrm{s})$ that is separately confirmed by the thermocouple measurements. Indeed, it might appear contradictory that the sound-speed change estimation is very far from its expected value with the inversion data producing a $97 \%$ variance reconstruction for travel-time fluctuations. This result finds explanation through the spatial resolution of the tomography data. Here the 4-mm size of the resistor is actually much smaller than the $\sim 50$ - $\mathrm{mm}$ wide sound-speed heterogeneity that is reconstructed from the acoustic measurements in the source-receiver plane. If the sound speed was summed over the expected size of the heat spot in Fig. 5, then a correct estimation of the sound-speed change is obtained. In other words, due to the poor spatial resolution in the range of the acoustic inversion (as specified in Sec. III, $\lambda_{r}=37 \mathrm{~mm}$ and $\lambda_{z}=5 \mathrm{~mm}$ were used as the correlation lengths in range and depth), the sound-speed change reconstruction is distributed over a larger heat spot than expected. Physically speaking, the spatial resolution of the acoustic tomography is limited by the size of the Fresnel zone associated with the TSK between the source-receiver arrays. Using vertical arrays, it is well known that the axial resolution of the diffraction pattern is significantly worse than the vertical resolution. In the far field, the lateral resolution at range $R$ of a vertical array (of aperture $D$ ) is classically defined as $\delta_{z} \sim \lambda R / D$ when the axial resolution is $\delta_{R} \sim 8 \lambda(R / D)^{2}$. Transposing these depth/range resolutions in the waveguide is complicated because the aperture of the vertical array should take into account the use of reverberation on the waveguide interfaces. Actually, $D / R$ should then be the effective angular aperture of the array in the waveguide. Assuming eigenray angles up to $\pm 14^{\circ}$, then $D / R \sim \tan \left(28^{\circ}\right)$ which finally gives $\delta_{z} \sim 3 \mathrm{~mm}$ and $\delta_{R} \sim 40 \mathrm{~mm}$ at $1 \mathrm{MHz}$. In conclusion, despite the use of high-angle eigenrays provided by the multipath propagation, the low-range resolution of the tomography result has a strong impact on the estimation of the sound-speed fluctuations.

Figure 7 shows the tomography data at the end of the heating process $\left(\tilde{t}=T_{0}+T\right)$, when most of the thermal plume 


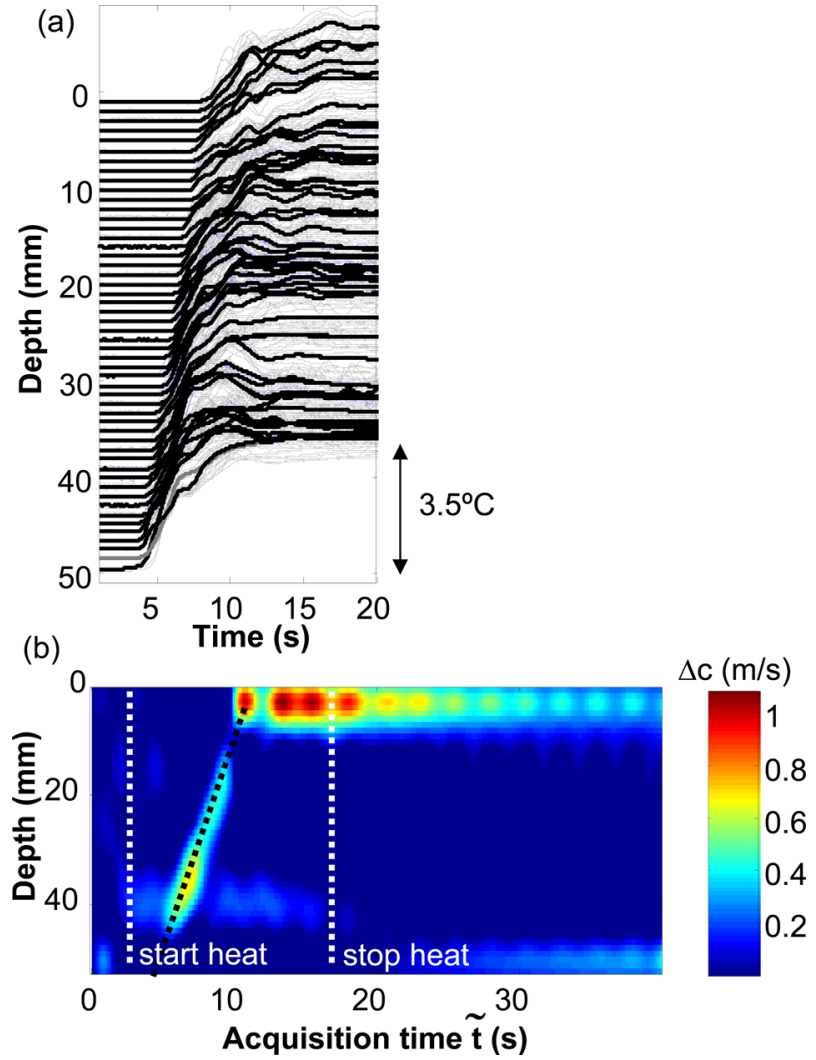

FIG. 6. (Color online) (a) Temperature probing of the thermal plume generated by the heating process using a thermocouple positioned every $1 \mathrm{~cm}$, from $z=50 \mathrm{~mm}$ (bottom) to $z=0$ (surface), in the waveguide. At each position, 10 heating cycles (with $T_{0}=2.5 \mathrm{~s}$ ) were performed (gray lines) from which an average depth-dependent temperature evolution is obtained (bold line). An average maximum of $3.5^{\circ} \mathrm{C}$ is reached, close to the thermal resistance. (b) Depth versus time evolution of the acoustic tomography at a range of $225 \mathrm{~mm}$ (position of the thermal resistance) using diffraction-based TSK at $3 \mathrm{MHz}$ and with DBF. The vertical convection of the thermal plume in the waveguide is clearly seen through the acoustic measurement at an average speed of $10 \mathrm{~mm} / \mathrm{s}$ (black dotted line). The white dotted lines indicate the start and the end of the heating process.

accumulates at the waveguide surface. The four panels illustrate the quality of the tomography inversion versus frequency (at 3 and $1 \mathrm{MHz}$ ), and also with respect to the forward model used. At $3 \mathrm{MHz}$, both ray theory and diffraction-based forward modeling allow satisfactory inversion in the waveguide although the diffraction-based TSK provides a better estimation of the sound-speed change. At $1 \mathrm{MHz}$, the difference between ray theory and Fresnel diffraction is more obvious. This result was expected because ray theory loses its validity at low frequencies. Note also that despite some noise in the inversion data, the sound-speed estimation is stronger at $1 \mathrm{MHz}$ than at $3 \mathrm{MHz}$ [Figs. 7(b) and 7(a)], due to a longer heating cycle $T(T=20 \mathrm{~s}$ at $1 \mathrm{MHz}$, and $T=15 \mathrm{~s}$ at $3 \mathrm{MHz}$ ).

This shallow water tomography analysis at the laboratory scale is finally concluded by the study of surface waves at the air-water interface during the heating process. The heating experiment described in the preceding text was repeated at $3 \mathrm{MHz}$ in the presence of small surface waves generated by the water filtration system inside the tank. Figure 3 shows the travel-time fluctuations extracted for two eigenrays after DBF. The effects of the surface waves on the travel-time fluctuations are clearly significant on eigenray 2 , the path of which interacts twice with the air-water interface. Note that the travel-time perturbations $\Delta \tau$ are only on the order of a few percent of the average period $T_{c}$. Note also that the accuracy of the travel-time perturbation measurements after DBF is at least one order of magnitude smaller $\left(\Delta \tau / T_{c}<0.1 \%\right)$ in this ultrasonic configuration. Comparing Figs. 3(b) and 3(c), it can be seen that the waves at the air-water interface induce similar travel-time perturbations after two surface reflections to the sound-speed change due to local heating. However, the dynamics of the surface waves and the heating phenomenon are different; this allows separation of the two by frequency filtering [Fig. 3(c)]. Focusing on the high-frequency surface-wave contribution only, the rms height of the surface waves $\sigma_{h}$ is inverted from the rms of the travel-time fluctuations. Indeed, in the Kirchhoff approximation (Williams et al., 2004), it is assumed for each eigenray:

$$
\sigma_{h}=\frac{c \sigma_{t}}{2 \sin \theta \sqrt{N}}
$$

where $\sigma_{t}$ is the rms of the travel-time fluctuations $\Delta \tau$ measured over the duration of the experiment, $\theta$ is the grazing angle, and $N$ is the number of surface reflections for each eigenray. Figure 8 shows the experimental values obtained for $\sigma_{h}$ using 1400 eigenrays with $N$ from 1 to 5 . The independence of $\sigma_{h}$ from $N$ is satisfactory. Note, however, that the $\sigma_{h}$ values are more dispersed for $N=1$; this corresponds to a low grazing angle for which the Kirchhoff approximation may no longer be valid at $3 \mathrm{MHz}$.

The value $\sigma_{h} \sim 20 \mu \mathrm{m}=\lambda / 25$ ( $\lambda=0.5 \mathrm{~mm}$ is the wavelength at $3 \mathrm{MHz}$ ) shows that the surface scattering strongly impacts on the travel-time measurements in the shallow water even for waveguides with small surface-wave amplitudes. Assuming that DBF provides travel-time perturbation measurements with high accuracy, shallow-water tomography can appear more viable at low frequency using diffraction-based TSK than at high frequency where surface scattering can dominate.

\section{DISCUSSION AND CONCLUSION}

In this section, the tomography results obtained at the laboratory scale are analyzed in the framework of the shallow-water tomography at the true ocean scale.

In shallow water, OAT has to compete with moored thermistor strings and conductivity-temperature-depth (CTD) bearing gliders that are technologies that provide higher resolution, lower variance estimates of the ocean sound speed. However, as shown at the ultrasonic scale, the unique advantage of OAT is to monitor a full slice of the ocean structure in real time, which should provide a spatial (range and depth) and temporal measurement of the dynamics of mixing layers in shallow water or internal waves in deep-water environments. On the other hand, a degraded range resolution is also observed at the ultrasonic scale that could be predicted a priori and that mostly depends on the 


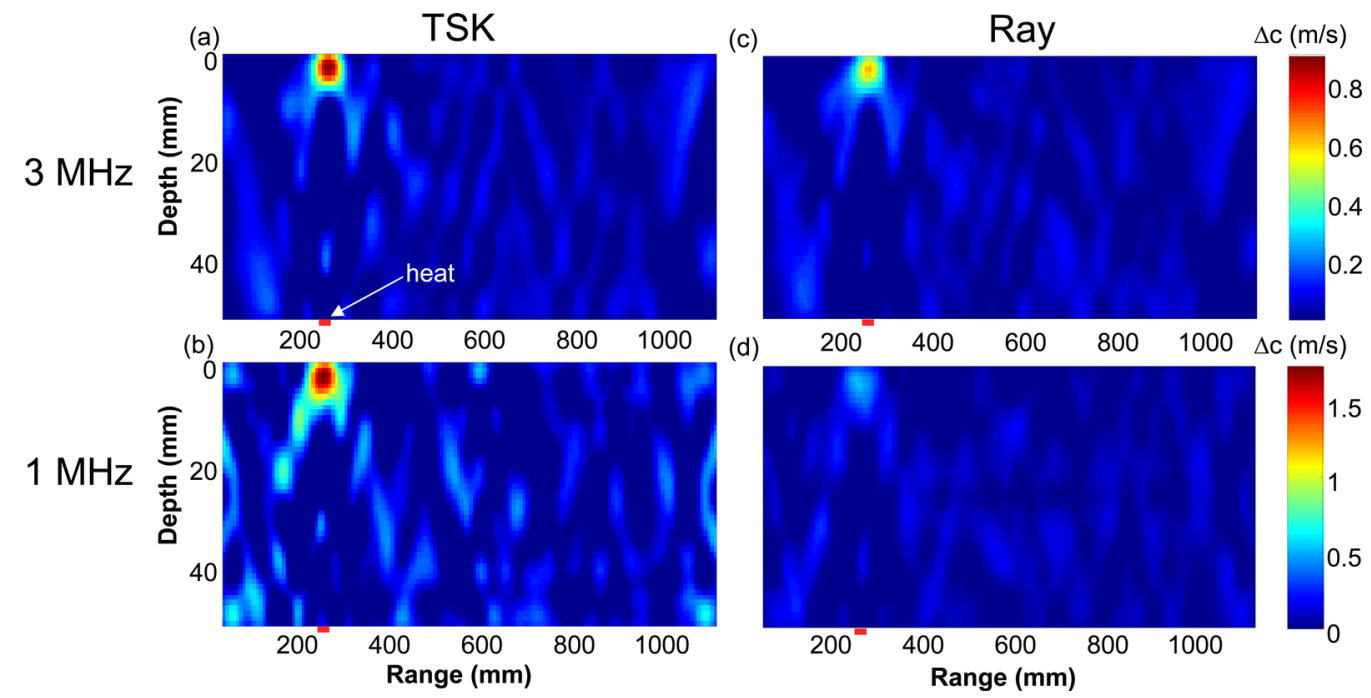

FIG. 7. (Color online) Experimental comparison of travel-time tomography data recorded at the frequencies of $3 \mathrm{MHz}$ (a) and (c) or $1 \mathrm{MHz}$ (b) and (d) and using a diffraction-based propagation model (a) and (b) or a ray-based approach (c) and (d). The sound-speed inversion at 3 and $1 \mathrm{MHz}$ corresponds to the end of the heating cycle $\left(\tilde{t}=T_{0}+T\right.$, with $T=15 \mathrm{~s}$ at $3 \mathrm{MHz}$ and $T=20 \mathrm{~s}$ at $\left.1 \mathrm{MHz}\right)$. As expected, tomography inversion using a ray model gives degraded results at the low frequency. The thermal resistance is located at a range of $225 \mathrm{~mm}$ as indicated by the white arrow (heat) in (a).

central frequency of the transducers and the source-receiver range (Fig. 4).

The combination of DBF and OAT is also a clear advantage because the tomography inversion benefits from the identification of a large number of acoustic eigenrays, which cover the whole acoustic waveguide. Such spatial coverage removes the eventual ambiguity in the location of the localized sound-speed perturbation. For better understanding, one may consider two eigenrays coming from the same source-receiver pair crossing twice in the water column. If a sound-speed heterogeneity is present at one crossing, both eigenrays would be subject to the same travel-time change but the acoustic inversion performed from these two eigenrays would not distinguish between the actual sound speed heterogeneity location and a phantom heterogeneity located at the other eigenray crossing. In a second step, if the source-receiver pair is now slightly shifted up and down in the water column in such a way that the two eigenrays paths do no longer both cross the sound speed heterogeneity, they may carry then different travel-time information. The phantom will tend to disappear if the inversion takes into account these modified eigenrays in addition to the previous ones.

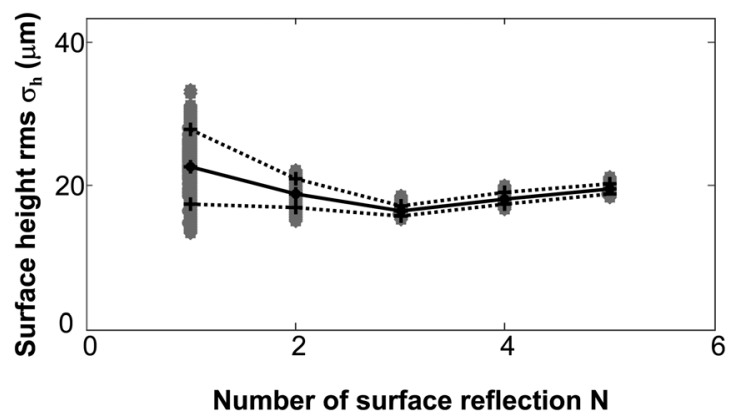

FIG. 8. Surface height rms $\sigma_{h}$ deduced from the high-frequency part of the travel-time fluctuations measured on the eigenrays with different numbers of surface reflections $(\mathrm{N})$. Gray stars, all of the DBF eigenrays; black line, the average $\sigma_{h}$; dotted lines, average $\sigma_{h} \pm$ standard deviation.
In the present paper, despite the symmetry in the source array-receiver array geometry, the use of $11 \times 11$ subarrays that span the whole water column on each side of the waveguide generates enough spatial diversity through the DBF eigenray extraction process to avoid the presence of hot-spot phantom in the tomography data (Fig. 5).

The use of diffraction-based TSK instead of the raybased approach is also an important step forward in OAT as can be seen from the finite-frequency results in Fig. 7. However, the calculation of the TSK can be time consuming, as it formally requires the broadband computation of the Green's function between every source and every receiver to all diffraction points in the waveguide. The computation time is significantly reduced when the TSK is calculated from the farfield diffraction pattern of the arrays, but this approximation leads to incorrect results at the bottom interface of the waveguide.

Finally, the strong effect of surface fluctuations at the air-water interface is observed in Fig. 3(c) on the travel-time perturbations due to a local sound-speed change in the waveguide volume. One way to tackle this major difficulty is to resolve the different time scales of the oceanic fluctuations. At the true scale of the shallow ocean, the time necessary to acquire the transfer matrix in transmission is only dependent on the number of sources and the time spread due to multipaths in the waveguide. Using a typical distance of $5 \mathrm{~km}$ between the source-receiver arrays and a critical angle of $10^{\circ}$, an average time spread of $50 \mathrm{~ms}$ can be expected at a few kilohertz (Roux et al., 2004), which means that the full transfer matrix can be acquired in $1.5 \mathrm{~s}$ for 30 -element source-receiver arrays. If a tomography experiment was performed with this repetition rate, it might be possible to resolve the dynamics of the ocean surface (the typical period of which is on the order of $7 \mathrm{~s}$ ). In such a case, low-pass filtering will separate the high-frequency surface-fluctuation effects from the low-frequency volume fluctuations on the travel-time measurements after DBF, resulting in the study 
of the main sources of environmental fluctuations in the waveguide, such as internal waves, array-element motion, unsteady currents, or heterogeneities in the water column.

In conclusion, the feasibility of OAT was experimentally demonstrated at the laboratory scale in a shallow-water configuration. The dynamics and spatial shape of a convecting thermal plume generated by a thermal resistor is clearly imaged through acoustic measurements. The tomography inversion results from the combination of the DBF algorithm performed between two source-receiver arrays and the forward model based on a finite-frequency approach. The DBF algorithm provides the separation and identification of a few thousand eigenrays from the data matrix, which ensures complete spatial coverage of the ultrasonic waveguide. The TSK applied to travel-time fluctuations after DBF takes advantage of the limited bandwidth of the acoustic signals. Compared to tomography data based on ray theory that are no longer relevant at low frequencies, this provides diffraction-limited spatial accuracy in range and depth. The spatial resolution of the tomography data is limited by the range resolution of the two vertical arrays on each side of the waveguide. When strong interactions with the rapidly fluctuating ocean surface are noticeable, acoustic acquisitions must be performed fast enough to separate the travel-time fluctuations associated with the ocean surface from the slow soundspeed variations in the ocean volume.

Apel, J., Badiey, M., Chiu, C.-S., Finette, S., Headrick, R., Kemp, J., Lynch, J., Newhall, A., Orr, M., Pasewark, B., Tielbuerger, D., Turgut, A., Heydt, K. V. D., and Wolf, S. (1997). "An overview of the 1995 SWARM shallow-water internal wave acoustic scattering experiment," IEEE J. Ocean. Eng. 22, 465-500.

Battle, D., Gerstoft, P., Hodgkiss, W., and Kuperman, W. A. (2004).

"Bayesian model selection applied to self-noise geoacoustic inversion," J. Acoust. Soc. Am. 116, 2043-2056.

Chiu, C-S., and Desaubies Y. (1987). "A planetary wave analysis using the acoustic and conventional arrays in the 1981 ocean tomography experiment," J. Phys. Oceanogr. 17, 1270-1287.

Cornuelle, B., Wunsch, C., Behringer, D., Birdsall, T., Brown, M., Heinmiller, R., Knox, R., Metzger, K., Munk, W., Spiesberger, J., Spindel, R., Webb, D., and Worcester, P. (1985). "Tomographic maps of the ocean mesoscale. I. Pure acoustics," J. Phys. Oceanogr. 15, 133-152.

Dahlen, F. A., Hung, S.-H., and Nolet, G. (2000). "Fréchet kernels for finitefreqeuncy traveltimes. I. Theory," Geophys. J. Int. 141, 157-174.

Dzieciuch, M., Worcester, P., and Munk, W. (2001). "Turning point filters: Analysis of sound propagation on a gyre scale," J. Acoust. Soc. Am. 110, 135-149.

Iturbe, I., Roux, P., Nicolas, B., Virieux, J., and Mars, J. (2009a). "Shallowwater acoustic tomography performed from a double beamforming algorithm,” IEEE J. Ocean. Eng. 34, 140-149.
Iturbe, I., Roux, P., Virieux, J., and Nicolas, B. (2009b). "Travel-time sensitivity kernels vs. diffraction pattern obtained through double beamforming in shallow water," J. Acoust. Soc. Am. 126, 713-720.

Lynch, J. F., Jin, G., Pawlowicz, R., Ray, D., Chiu, C.-S., Miller, J. H., Bourke, R. H., Parsons, A. R., Plueddemann, A. J., and Muench, R. (1996). "Acoustic travel-time perturbations due to shallow-water internal waves and internal tides in the Barents Sea Polar Front: Theory and experiment," J. Acoust. Soc. Am. 99, 803-821.

Marquering, H., Dahlen, F. A., and Nolet, G. (1999). "Three-dimensional sensitivity kernels for finite-frequency travel times: The banana-doughnut paradox," Geophys. J. Int. 137, 805-815.

Munk, W., Worcester, P., and Wunsch, C. (1995). Ocean Acoustic Tomography (Cambridge University Press, London), pp. 38-92.

Munk, W., and Wunsh, C. (1979). "Ocean acoustic tomography: A scheme for large scale monitoring," Deep-Sea Res. 26, 123-161.

Munk, W., and Wunsch, C. (1983). "Ocean acoustic tomography: Rays and modes," Rev. Geophys. Space Phys. 21, 777-793.

North Pacific Acoustic Laboratory (NPAL), Experiments were designed to study coherence of acoustic signals propagating long distances in the deep ocean. The acoustic source was $3000 \mathrm{~km}$ from the receiver arrays. Their array technology is the same as that used in the Acoustic Thermometry of the Ocean Climate experiments [ATOC Consortium (1998). "Ocean climate change: Comparison of acoustic tomography, satellite altimetry and modeling," Science 281, 1327-1332].

Rajan, S.-D., Lynch, J.-F., and Frisk, G.-V. (1987). "Perturbative inversion methods for obtaining bottom geoacoustic parameters in shallow water," J. Acoust. Soc. Am. 82, 998-1017.

Rodriguez, O., and Jesus, S. (2000). "Physical limitations of travel-timebased shallow-water tomography," J. Acoust. Soc. Am. 108, 2816-2822.

Roux, P., Cornuelle, B. D., Kuperman, W. A., and Hodgkiss, W. S. (2008). "The structure of ray-like arrivals in a shallow water waveguide," J. Acoust. Soc. Am. 124, 3430-3439.

Roux, P., Kuperman, W. A., Hodgkiss, W. S., Song, H. C., Akal, T., and Stevenson, M. (2004). "A non-reciprocal implementation of time reversal in the ocean," J. Acoust. Soc. Am. 116, 1009-1015.

Skarsoulis, E. K., and Cornuelle, B. D. (2004). "Travel-time sensitivity kernels in ocean acoustic tomography," J. Acoust. Soc. Am. 116, 227238.

Taniguchi, N., Kaneko, A., Yuan, Y., Gohda, N., Chen, H., Liao, G., Yang, C., Minamidate, M., Adityawarman, Y., Zhu, X., and Lin, J. (2010). "Long-term acoustic tomography measurement of ocean currents at the northern part of the Luzon Strait," Geophys. Res. Lett. 37, L07601.

Tarantola, A. (1984). "Inversion of seismic reflection data in the acoustic approximation," Geophysics 49, 1259-1266.

Tarantola, A. (1987). Inverse Problem Theory: Methods for Data Fitting and Model Parameter Estimation (Elsevier, New York), pp. 68-71.

Tromp, J., Tape, C., and Liu, Q. (2005). "Seismic tomography, adjoint methods, time reversal, and banana-donut kernels," Geophys. J. Int. 160, 195-221.

Williams, K. L., Thorsos, E. I., and Elam, W. T. (2004). "Examination of coherent surface reflection coefficient (CSRC) approximations in shallow water propagation," J. Acoust. Soc. Am. 116, 1975-1984.

Woodward, M. J. (1992). "Wave-equation tomography," Geophysics 57, $15-26$.

Worcester, P. F., Spindel, R. C., and Howe, B. M. (1985). "Reciprocal acoustic transmissions: Instrumentation for mesoscale monitoring of ocean currents," IEEE J. Ocean. Eng. 10, 123-137. 